\title{
LA CORRIENTE DE CROMWELL DURANTE EL FENÓMENO LA NIÑA DE 1996 Y EL FENÓMENO EL NIÑO DE 1997, SOBRE LA CUENCA DEL PACIFICO COLOMBIANO.
}

\section{Alex R. Pineda D.}

\section{RESUMEN}

Se han estudiado en conjunto los parámetros térmicos, halinos y de dirección geostrófica, de una de las corrientes más largas del mundo, que incide sobre el Pacifico sur colombiano, como es la corriente de Cromwell, durante dos épocas anómalas, como fueron el fenómeno de "La Niña" de 1996 y el fenómeno de "El Niño" de 1997. Se determinó que la corriente de Cromwell, se presentó durante estas épocas anómalas. Durante la época fría se observó ligeramente más superficial entre los 75 y 200 metros de profundidad, con salinidades de 34.9 o/00 y durante la épisca cálida un poco más profunda entre los 100 y 300 metros, con condiciones halinas de 35 a 35.1 psu. Se podría decir que bajo condiciones anómalas positivas, la corriente se registra menos intensa, sobre el área de estudio.

\begin{abstract}
The current of Cromwell is one of world's largest oceanic current which influences the Colombian South Pacific. The present work studied the thermo-haline parameters and the geostrophic patterns during two air-sea interactions events as "La Niña" (1996) and "El Niño" (1997). It has been determined that the Current of Cromwell was present during both phenomena. During the cold event (La Niña) it was slightly upper, between $75-200$ meters depth with salinities of $34.90 / 00$ and for the warm event (EI Niño) it was deeper between $75-300$ meters with haline conditions about $35-35.1 \mathrm{o} / 00$. It is possible to conclude that under positive anomaly conditions or cold event, the Current is less intense at the Colombian South Pacific than in the negative one.
\end{abstract}




\section{INTRODUCCIÓN}

La corriente de Cromwell o subcorriente Ecuatorial, atraviesa el Océano Pacífico desde Nueva guinea hasta las costas de Sudamérica. Su longitud está cerca a los $16.000 \mathrm{~km}$., incidiendo sobre las condiciones higrológicas y biológicas del sector costero del Océano Pacífico Oriental. la corriente de Cromwell es una corriente subsuperficial, que se dirige hacia el este, en la zona ecuatorial de los $2^{\circ}$, tanto de latitud norte como de latitud sur. La corriente normalmente se encuentra y se desplaza entre las capas de los 200 300 metros, a lo largo clel Pacífico Ecuatorial y al acercarse a las Islas Galapagos y el continente, la corriente de Cromwell se localiza en la capa de 80 a 100 metros de profundidad (Bubnov).

El evento cálido de El Niño, se inicia con el enfriamiento de las aguas en el Pacífico ecuatorial del este, que se produce por la intensificación de los vientos alisios provenientes de la Antártida, generando la corriente marina fría de Humboldt sobre el sector oceánico de Chile y Perú. Los vientos alisios al aumentar, empujan las aguas frías hacia el Pacífico Ecuatorial Oriental, intensificando la Corriente Ecuatorial y desplazando grandes cantidades de agua hacia el Pacífico Ecuatorial Occidental, haciendo que el nivel del mar ascienda en esta zona, y la termoclina se profundice. Esta etapa fría puede durar varios meses.

Después de esta fase fría, se observa la disminución de los vientos alisios del este, debilitando la corriente de Humboldt, cesando el empuje de agua hacia el Pacifico central y occidental. Al suceder esto, la masa de agua que se encuentra en el Pacífico Occidental se desplaza hacia el Pacifico Oriental , y después hacia las costas suramericanas, aumentando considerablemente la temperatura del agua y el nivel del mar; este proceso es lento. La masa de agua desplaza consigo la nubosidad hacia el Pacífico central y oriental, produciendo lluvias. Así mismo, el viento invierte su rumbo tomando dirección oeste - este, alterando el clima sobre el Océano Pacífico.

La presente investigación tiene por objeto el estudio y comportamiento de la corriente de Cromwell durante el evento frío del Pacífico de 1996 y el evento cálido del Pacífico de 1997. Se analizará su comportamiento a partir de las características térmicas y halinas, se indicará la capa en la cual se transporta la corriente sobre el Pacífico Sur Colombiano, y por último se utilizará un modelo dinámico, que dará la idea de la circulación de la corriente en el área, donde esta incide. En este estudio se consideraron tres perfiles perpendiculares a la costa, dos de estos sobre los puertos de Buenaventura y Tumaco y uno sobre el perfil de Isla Gorgona. Se analizaron los datos físicos (Temperatura y Salinidad) hasta una profundidad de 500 metros, para determinar la presencia de la corriente de Cromwell en el Pacífico sur colombiano. Así mismo, utilizando un modelo dinámico, se da una idea de la circulación de la corriente, en los horizontes 100 y 200 metros, profundidades en las cuales incide la corriente de Cromwell en el Pacífico sur colombiano.

\section{MATERIALES Y MÉTODOS}

\section{Área de Estudio}

El área de estudio comprende la zona sur del Pacífico Colombiano, tomando como referencia termohalina los perfiles de Tumaco, Isla Gorgona y Buenaventura, identificados en el presente documento de la siguiente manera :

1. Perfil perpendicular a Tumaco localizado desde los $79^{\circ}$ a $82^{\circ}$ de longitud $W$ y latitud $02^{\circ} \mathrm{N}$, con las siguientes estaciones 33,41 , $49,57,65,73$ y 81 . 
2. Perfil perpendicular a la Isla Gorgona, comprendida entre la Longitud $81^{\circ} 30^{\prime} \mathrm{W}$ a $79^{\circ}$ $\mathrm{W}, \mathrm{y}$ Latitud $03^{\circ} \mathrm{N}$, con las siguientes estaciones $16,24,31,39,47,55,63,71$ y 79 .

3. Perfil perpendicular entre Buenaventura Isla Malpelo, limitado por la Latitud $04^{\circ} \mathrm{N}$ entre los $82^{\circ}$ y $78^{\circ} \mathrm{W}$, con las siguientes estaciones $6,14,22,29,37,45,53,61,69$ y 77.

\section{Metodología}

Los datos utilizados en el presente estudio fueron obtenidos por el Centro Control Contaminación del Pacífico abordo del B/O ARC Malpelo, durante los cruceros oceanográficos PACIFICO XXV - ERFEN XXIII / Junio-96, PACIFICO XXVI - ERFEN XXIV / Octubre-96, PACIFICO XXVII - ERFEN XXV / Mayo-97, y PACIFICO XXVIII - ERFEN XXVI / Nov-Dic/ 97.

Se tomó la información hidrológica básica de temperatura y salinidad del mar, tanto en superficie como en profundidad. Se analizó el comportamiento de la corriente de Cromwell, con el fin de observar las características de los indices termohalinos en condiciones de un fenómeno frío ( La Niña ) y cálido ( El Niño ), lo cual resulta interesante para conocer su comportamiento en cada una de estas épocas anómalas. Para el efecto se utilizó la distribución vertical de la temperatura y salinidad hasta una profundidad de 500 metros; y se elaboraron gráficas horizontales de la topografía dinámica a los 100 y 200 metros de profundidad.

\section{Cálculo de la Topografía Dinámica}

Los resultados de la dirección de la corriente fueron obtenidos empleando el método de las corrientes de densidad elaborada por Helland-Hansen, basándose en la teoría de la circulación de Bjerknes.
El método supone o parte de que la variación de la presión (dp) en el mar es proporcional a la variación del peso de la columna de agua, es decir,

$$
\mathrm{d}=\rho^{\mathrm{o}^{*} \mathrm{~g} * \mathrm{~d}}
$$

Teniendo en cuenta que la densidad del agua $\left(\rho^{0}\right)$ es un valor inverso al volumen especifico ( $\underline{\text { Ve }}$, es decir $\rho^{0=1}$ śepuede escribir,

$$
V e^{*} d p=-g * d z
$$

Integrando esta expresión, obtendremos

$$
\int_{p}^{P_{0}} V e * d p=-\int_{z}^{0} g d z=\mathrm{g} * \mathrm{z}=\mathrm{D},
$$

donde $z$ es la distancia entre las superficies isobáricas. Sustituimos la integral por la Suma

$$
\int_{P}^{P_{0}} V e^{*} d p=\sum_{P}^{P_{0}} V e^{*} \Delta p=\mathrm{D} .
$$

Al calcular las alturas dinámicas se toma no el volumen específico real, sino el convencional. En vez de utilizar la densidad del agua de mar, es más conveniente valerse de la magnitud inversa, llamada volumen especifico del agua del mar. Debido a que el volumen específico siempre es mayor de 0.9 , pero menor de 1.0 , para abreviar la escritura ha sido introducido, el concepto de volumen específico convencional $(\mathrm{Vt})$, determinable por la relación

$$
\mathrm{Vt}=(\mathrm{Ve}-0.9) * 10^{3}
$$

De donde

$$
\mathrm{Ve}=\mathrm{Vt} * 10^{-3}+0.9
$$

Y la fórmula será:

$$
\mathrm{D}=\sum_{P}^{P_{0}} V t * 10-{ }^{3} * \Delta p+\sum_{P}^{P_{0}} 0.9 * \Delta p
$$

Al calcular la corriente, se determinan las diferencias de las alturas dinámicas entre las superficies isobáricas, se puede despreciar el segundo sumando, y la fórmula será : 


$$
\Delta D=\sum_{P}^{P_{0}} V t * 10-3 * \Delta p
$$

Al trazar las cartas de la circulación por el método dinámico, es muy importante saber escoger la superficie isobárica inicial, a partir de la cual se cuentan las alturas dinámicas, la así llamada superficie cero. La elección de la superficie cero se hace habitualmente por métodos indirectos. Existen varios métodos para determinar la posición de la superficie cero en el océano. Consideremos algunos de ellos:

- Dietrich, propuso considerar como superficie cero la superficie con contenido mínimo de oxígeno en la profundidad.

- Hidaka considera que se puede determinar partiendo del cálculo de la difusión de las sales a diferentes profundidades. La capa donde ésta difusión es mínima es la que propone adoptar como superficie cero.

- Parr supone que el movimiento de las aguas transcurre a lo largo de las superficies isopícnicas. Por eso, con velocidad mínima de la corriente no existe inclinación de las superficies isopicnicas y la distancia entre ellas debe ser permanente.

- Defant propuso un método utilizando sólo los datos referentes a las alturas dinámicas de las superficies isobáricas. El méto- do consiste en la determinación de las diferencias de las alturas dinámicas entre estaciones oceanográficas vecinas.

Con la información que se posee, se determinó la superficie cero en el horizonte donde se presentó la concentración mínima de oxígeno, la cual se presentó a una profundidad promedio de 500 metros, en el Pacífico Sur Colombiano. La profundidad en metros fue considerada numéricamente igual a la presión en decibares ( $\mathrm{db}$ ). Para el comportamiento de la circulación se elaboraron gráficas de la topografía dinámica, para los horizontes 100 y 200 metros respectivamente.

\section{Régimen de la Circulación en Condi- ciones Normales en la Cuenca del Pacífico Colombiano}

La figura No.1, corresponde al régimen de circulación de la corriente de Cromwell sobre la Cuenca del Pacífico Colombiano, en donde se observa que la subcorriente hace su ingreso al área de estudio entre los $1^{\circ} \mathrm{N}$ y $2^{\circ} 30^{\prime} \mathrm{N}$, perfil de la frontera con el Ecuador, para dirigirse o desplazarse sobre todo el perfil de Isla Gorgona y llegar al perfil de Buenaventua. Entre este perfil $\left(4^{\circ} \mathrm{N}\right)$ y los

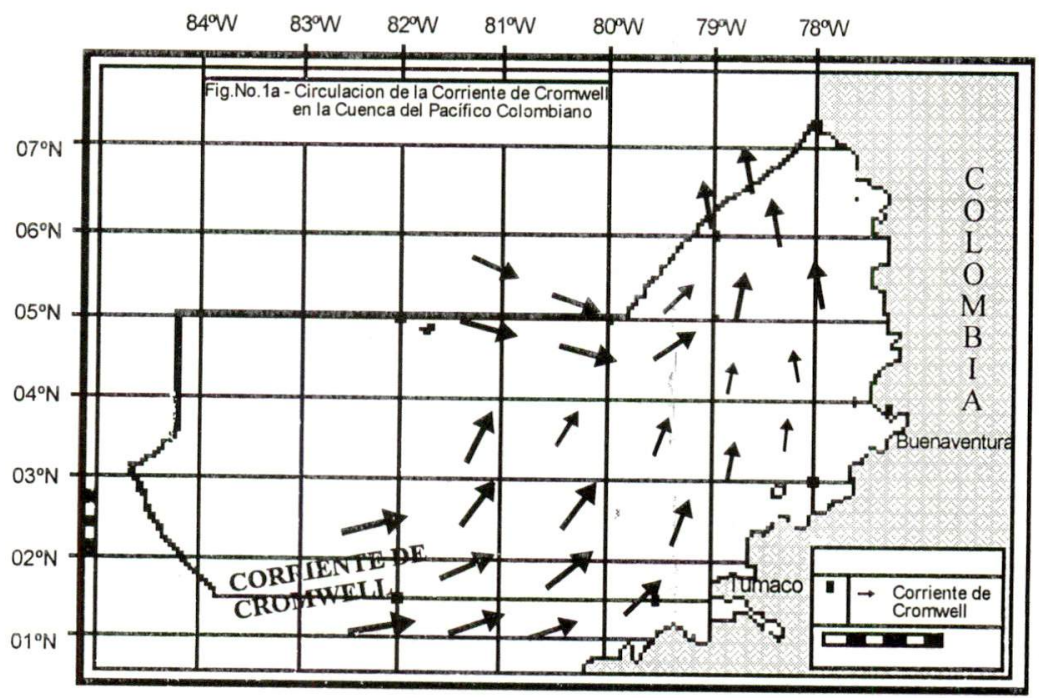

Fig $N^{\circ} 1$ 
$5^{\circ} \mathrm{N}$, la subcorriente se debilita al hacer contacto con las corrientes que se dirigen de Centroamérica y hacen su ingreso sobre el área norte, donde se mezclan y forman una circulación ciclónica sobre estos perfiles.

\section{RESULTADOS}

\section{Comportamiento Termohalino durante La Niña}

\section{Junio de 1996}

\section{Perfil de Tumaco:}

Entre las características relacionadas con la corriente de Cromwell, se tiene un indice de temperatura de agua ecuatorial de $13^{\circ} \mathrm{C}$ a $15^{\circ} \mathrm{C}$ y un núcleo de altas salinidades $(34.9$ a 35.0 o/oo). El índice térmico es notable por la gran separación de las isotermas e isopicnas justo bajo la termoclina (Lucero, Cornejo - Rodríguez, 1990).

Durante esta época en el perfil de Tumaco, ya se sentían las manifestaciones frías del evento de La Niña en el Pacifico Colombiano (Fig.No.2), y se observaba a la corriente no muy bien definida, sobre el área de estudio. Los índices térmicos no concuerdan con los halinos, ya que según la figura No.3, la corriente se encuentra muy profunda, por debajo de los 300 metros. Esto se debe posiblemente al cambio de las condiciones normales a frías.

Perfil de Isla Gorgona:

Sobre este perfil, la capa superficial indicaba un enfriamiento con temperaturas bajas y una termoclina superficial, lo cual evidenciaba la presencia del evento frío. Las características térmicas y la distribución de la salinidad (Figs. No.4 y 5), denotaban claramente la capa de alta salinidad (34.9 o/oo), localizando la corriente en los niveles de 50 a 250 metros.

\section{Perfil de Buenaventura:}

A nivel superficial, el comportamiento térmico presenta una conglomeración de las isotermas hasta los 100 metros, con temperaturas bajas, por el orden de $25^{\circ}$ a $18^{\circ} \mathrm{C}$. Por debajo de la termoclina se desplaza la corriente (Fig.No.6). Así mismo, el comportamiento halino, muestra una baja salinidad a nivel superficial sobre el sector costero (Estaciones $6-11-22$ ), impidiendo el afloramiento de la corriente, localizándose ésta entre los 75 y 200 metros de profundidad, presentándose como una lengua de agua salina de 34.9 o/0o (Fig.No.7).

\section{EVOLUCION DE LA CORRIENTE DE CROMWELL DURANTE MAYO DE 1996.}

Fig.No.2- Perfil de Tumaco Temperatura del Agua

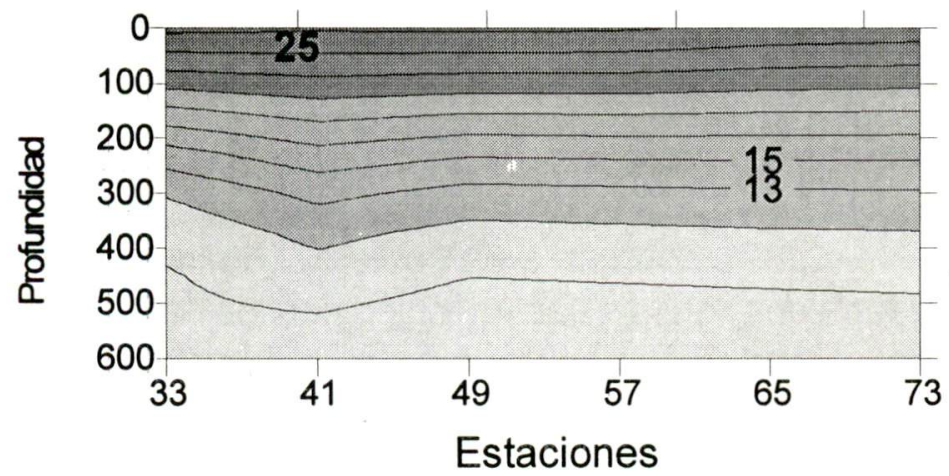

Fig.No. 3 - Perfil de Tumaco Salinidad del Agua

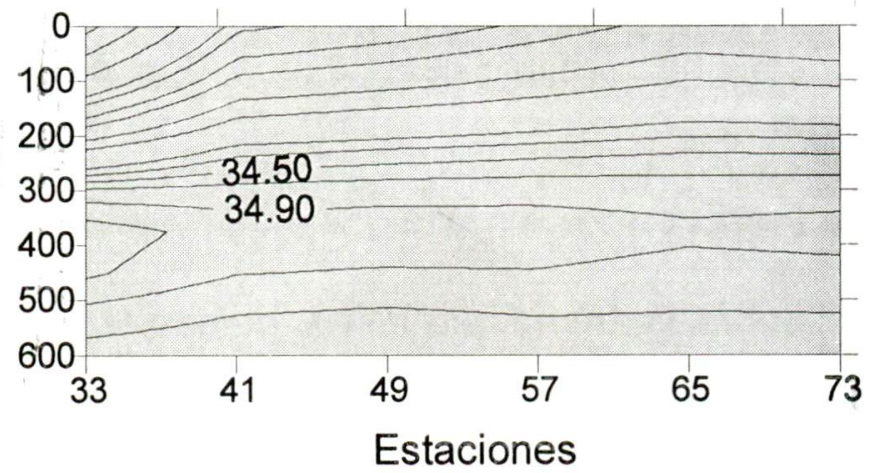


Fig.No.4- Perfil Isla Gorgona Temperatura del Agua Fig.No.5- Perfil de Gorgona Salinidad del Agua
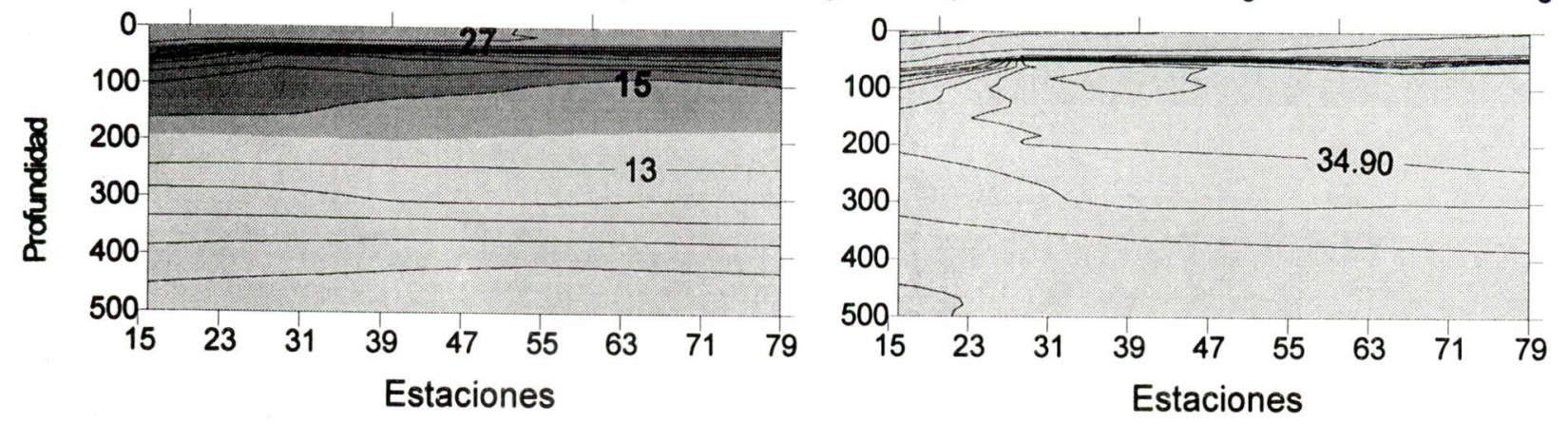

Fig.No.6 - Perfil de Buenaventura Temperatura del Agurag.No.7 - Perfil de Buenaventura Salinidad del Agua
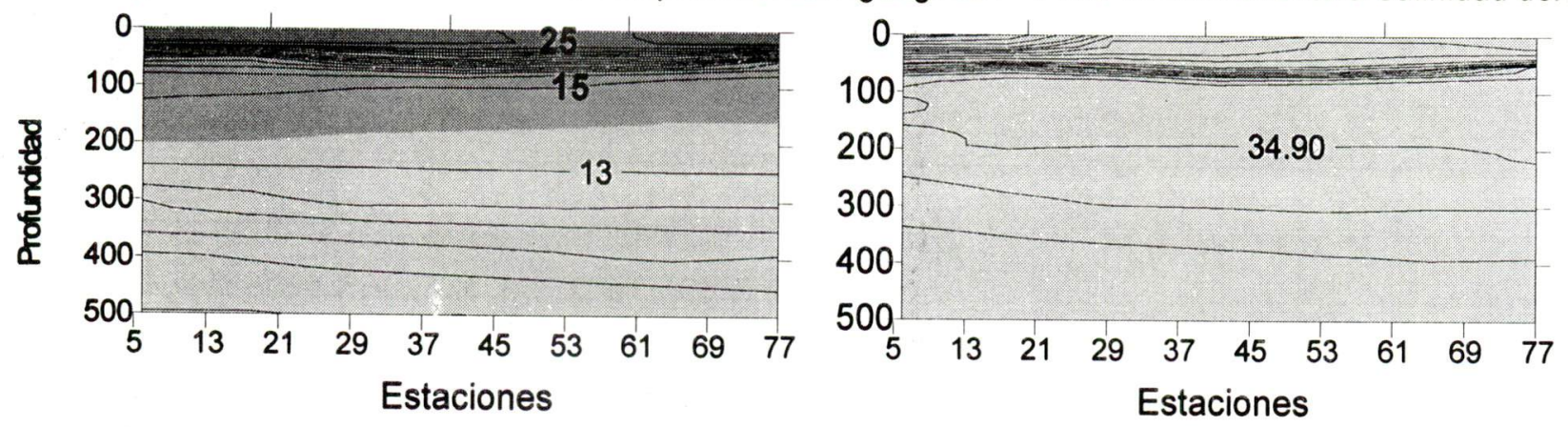

\section{Octubre de 1996.}

\section{Perfil de Tumaco}

Para este periodo se acentuaban un poco más las condiciones anómalas negativas del evento: la termoclina se encontró superficial en la capa de 25 a 40 metros, con temperaturas de $25^{\circ}$ a $17^{\circ} \mathrm{C}$. La temperatura superficial del mar no supero los $26{ }^{\circ} \mathrm{C}$ y la subcorriente se acercó a la superficie sobre el sector costero (Estaciones 33 y 41 ), estando entre los 50 y 200 metros. A nivel oceánico estuvo entre los 75 y 200 metros. El comportamiento halino es muy similar al térmico, registrándose a la misma profundidad, con una salinidad de 34.9 o/oo (Figs.No.8 y 9).

\section{Perfil de Isla Gorgona.}

Sobre este perfil no difiere demasiado a lo registrado en el perfil de Tumaco. La subcorriente se presenta con sus índices termo-halinos entre los 75 y 200 metros de pro-

fundidad. Los valores de temperatura típicos de la corriente $\left(13^{\circ}\right.$ a $\left.15^{\circ} \mathrm{C}\right)$, ubican muy bien a la subcorriente sobre este perfil. La figura No.11, presenta la entrada de una bolsa de agua salina típica de la subcorriente (34.9 o/ oo), la cual se debilita a medida que se acerca a la parte costera.

\section{Perfil de Buenaventura.}

Dúrante octubre de 1996, se evidenció la presencia de la corriente de Cromwell, sobre todo el Pacífico sur colombiano, sin llegar a diferir mucho entre los perfiles de Tumaco a Buenaventura. En este perfil se observa la entrada de la bolsa de agua salina (34.9 o/oo), un poço más profunda sobre el sector oceánico (estaciones 53 y 61), hacia los 250 metros, que a medida que se desplaza hacia la costa disminuye su espesor (75 a 200 metros), y se debilita sobre el área costera. Los índices térmicos concuerdan muy bien con los halinos y ubican a la subcorriente en la capa de los 75 a 200 metros de profundidad (Figs. No. 12 y 13). 


\section{EVOLUCION DE LA CORRIENTE DE CROMWELL DURANTE OCTUBRE DE 1996}

Fig.No.8 - Perfil de Tumaco Temperatura del Agua Fig.No.9 - Perfil de Tumaco Salinidad del Agua

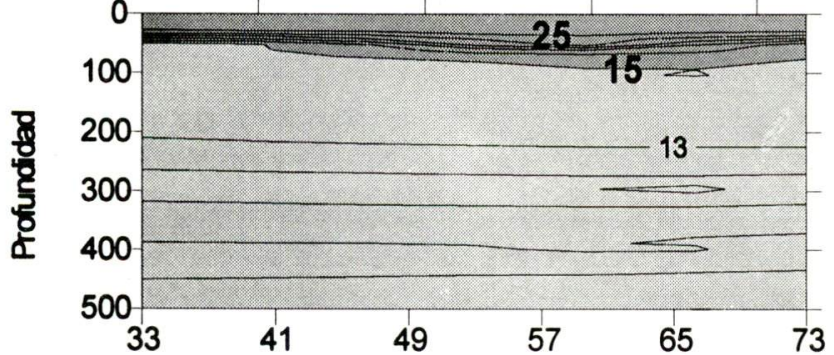

Estaciones

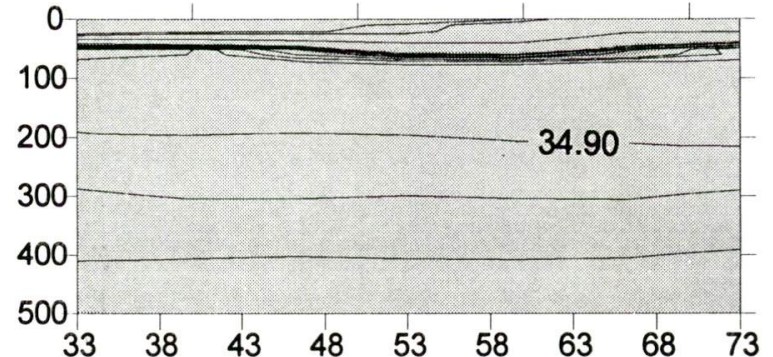

Estaciones

Fig.No.10 - Perfil de Gorgona Temperatura del Agua Fig.No.11 - Perfil de Gorgona Salinidad del Agua
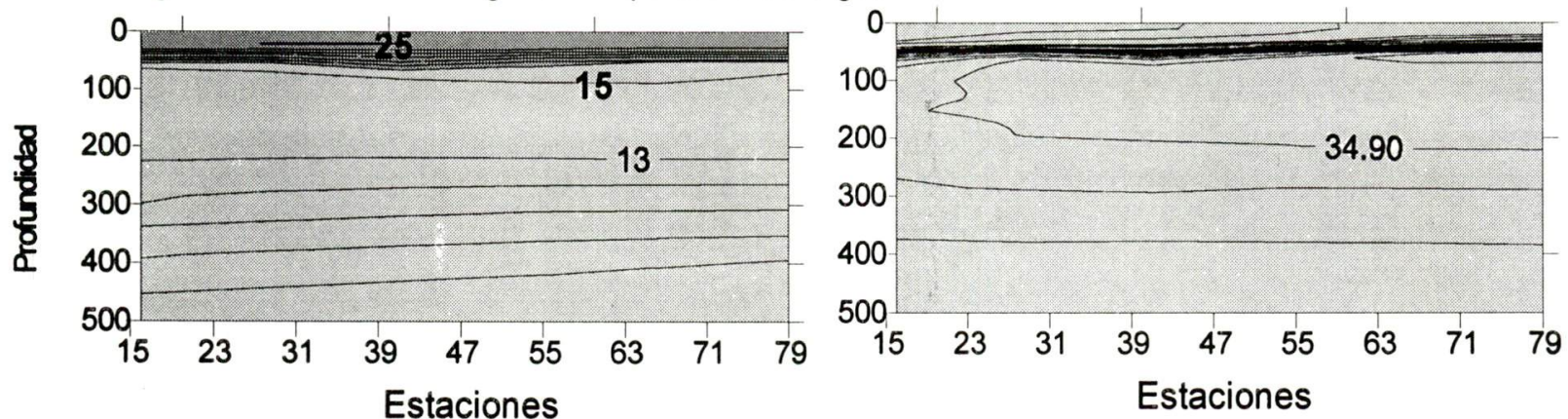

Fig.No.12 - Perfil de Buenaventura Temperatura del AğiggNo.13 - Perfil de Buenaventura Salinidad del Agua

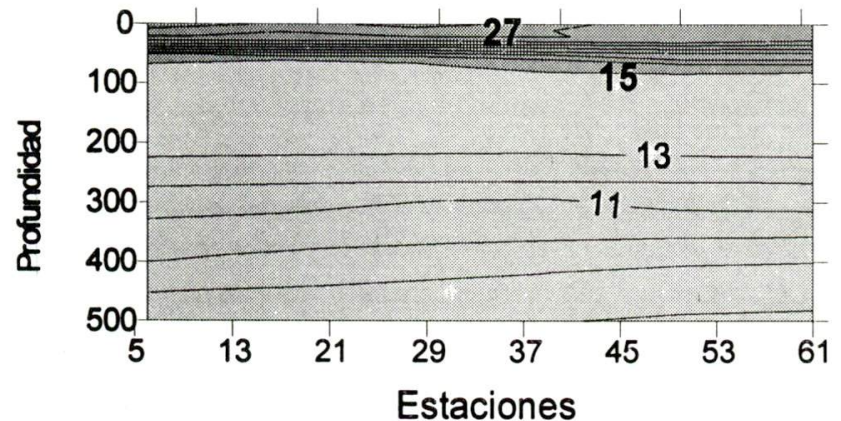

Comportamiento Termohalino durante El Niño.

\section{Mayo de 1997}

\section{Perfil de Tumaco.}

En esta época, el evento cálido del Pacífico comenzaba ha hacer notoria su presencia sobre aguas Colombianas, presentando temperaturas superficiales superiores a $28^{\circ} \mathrm{C}, \mathrm{y}$ una mayor profundización de la termoclina (60 metros). Así mismo, la subcorriente se

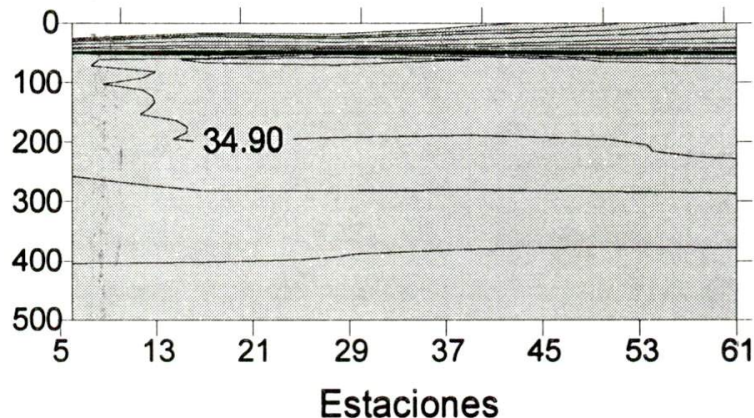

registra un poco profunda con sus índices termohalinos, estando en los niveles de 60 a 330 metros, aproximadamente. Entre los 85 y 180 metros de profundidad se observa una bolsa de agua salina típica de la corriente de Cromwell con un valor de 35 o/oo, la cual es recubierta por una lengua de agua salina de 34.9 o/oo, entre los 75 y 300 metros (Figs. No. 14 y 15).

Perfil de Isla Gorgona.

La figura No.17, de la salinidad del agua, pre- 
senta un núcleo salino de $35 \mathrm{psu}$, abarcando gran parte del área de estudio, entre las estaciones 39 a 79 . Este núcleo es recubierto por la isohalina 34.9 psu, típica de la corriente de Cromwell, ocupando las capas de 50 a 300 metros. A medida que la subcorriente se desplaza hacia la costa disminuye su espesor.

Sobre las estaciones 16 y 24 las salinidades son mucho menores estando por el orden de 29 a $31 \mathrm{psu}$. La temperatura del agua presenta una capa cálida de 75 metros sobre las estaciones más cercanas a la costa (16$24-31$ ), por el orden de $28^{\circ}$ a $23^{\circ} \mathrm{C}$, y los indices térmicos registran a la subcorriente

desplazándose en la capa de 200 a 300 metros (Fig.No.16).

Perfil de Buenaventura.

La gráfica No.18, de la temperatura del agua, muestra condiciones térmicas altas a nivel costero con oscilaciones entre 27 y $29^{\circ} \mathrm{C}$, la termoclina se localizó por debajo de los 100 metros de profundidad. Las temperaturas $13^{\circ}$ y $15^{\circ} \mathrm{C}$; indices de la subcooriente, se comportan muy similares al del perfil de Gorgona. La gráfica de la salinidad (Fig.No.19) registra el núcleo salino (35 o/oo), de menor tamaño al observado en los dos perfiles anteriores. La isohalina 34.9 o/oo, se desplaza entre los niveles de 100 y 300 metros, disminuyendo su grosor a nivel costero, por las bajas salinidades que se presentan, debido al aporte de los ríos sobre el área, que a nivel superficial presenta valores menores de 28 o/oo.

EVOLUCION DE LA CORRIENTE DE CROMWELL DURANTE MAYO DE 1997

Fig.No.14 - Perfil de Tumaco Temperatura del Agua Fig.No.15 - Perfil de Tumaco Salinidad del Agua
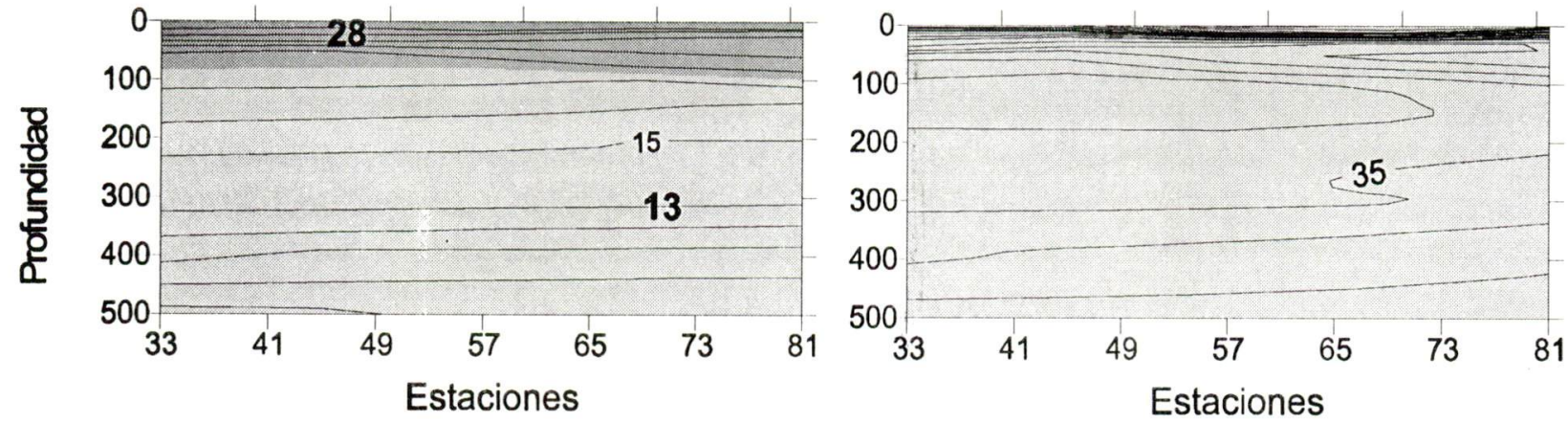

Fig.No.16 - Perfil de Gorgona Temperatura del Agua

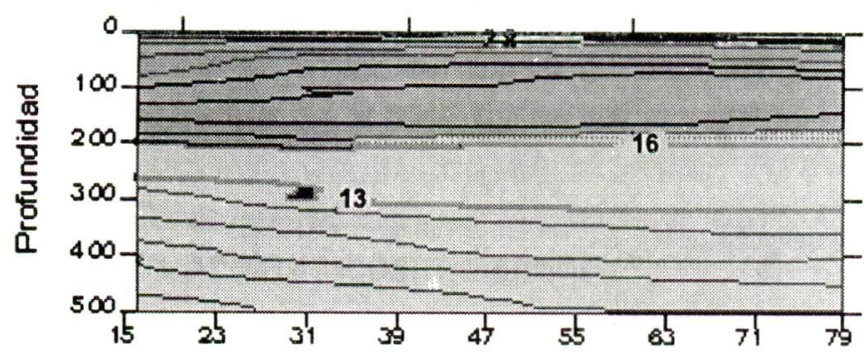

Fig.No.17 - Peril de Gorgona Salinidad del Agua

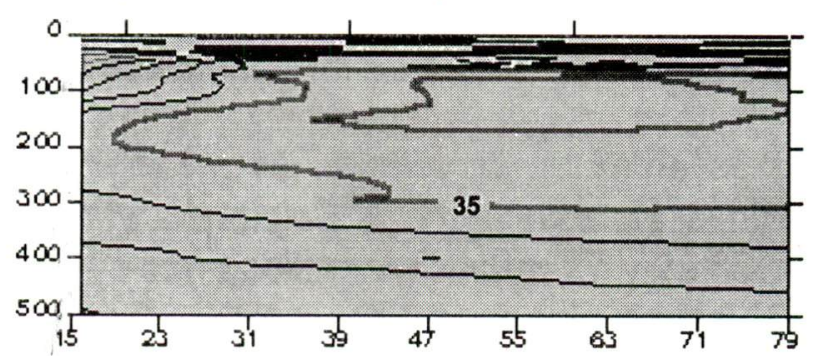

Estaciones

Estaciones

Fig. No.18-Peril de Buenaventura Temperatura del Agua

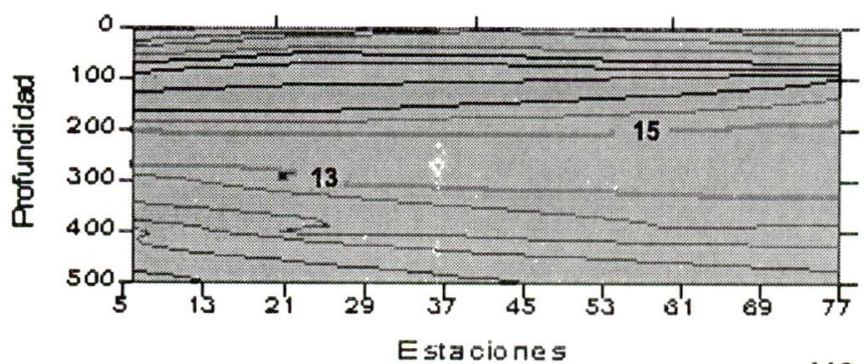

Fig.No.19-Peril de Guenaventura Salinidad del Agua

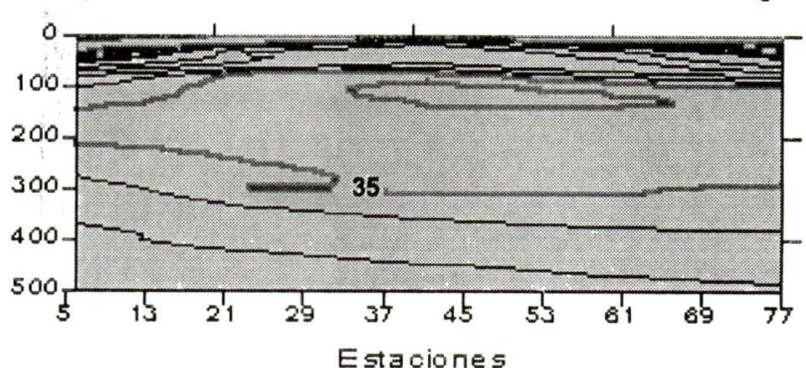




\section{Diciembre de 1997}

\section{Perfil de Tumaco:}

Durante esta época el evento cálido presentaba sus temperaturas más altas, con un promedio de $29^{\circ} \mathrm{C}$. La termoclina se ubicaba por debajo de los 125 metros, siendo para el área muy profunda. La corriente de Cromwell mostraba los índices térmicos en una capa de 50 metros (250 a 300 metros), esto como consecuencia del desplazamiento de una capa cálida superficial del Pacífico ecuatorial oriental, haciendo que la corriente se profundizara. Los indices halinos se registraban en un grosor aproximado de 200 a 250 metros. A nivel oceánico se ubicó entre las profundidades de 125 a 400 metros, y a nivel costero entre 125 y 325 metros (Fig.No.21), con salinidades de 35 o/oo.

\section{Perfil de Isla Gorgona:}

Sobre el perfil de Isla Gorgona, las características son similares al de Tumaco, observándose el calentamiento de sus aguas en una capa de 100 metros. La termoclina se ubicó por debajo de los 150 metros. Los índices térmicos de la subcorriente la ubican entre los 250 y 300 metros de profundidad. EI comportamiento de la salinidad (Fig.No.23) muestra a la subcorriente desplazándose entre los 100 y 300 metros de profundidad. EI comportamiento termohalino a nivel superficial, impide que la corriente suba y se desplace en la capa de transición térmica.

\section{Perfil de Buenaventura:}

Sobre este perfil, las condiciones térmicas presentan temperaturas superiores a $28.5^{\circ} \mathrm{C}$ a nivel superficial. La capa de transición térmica se ubico a una profundidad aproximada de 130 metros. Los indices térmicos muestran las mismas caraterísticas que en los perfiles de Tumaco y Gorgona. La salinidad presento una bolsa de agua salina de $35 \mathrm{psu}$, desplazándose sobre los 100 y 200 metros (Fig.No.25), esta bolsa es recubierta por la isohalina $34.8 \mathrm{psu}$, típica de la subcorriente ecuatorial.

\section{EVOLUCION DE LA CORRIENTE DE CROMWELL DURANTE DICIEMBRE DE 1997}

Fig.No.20 - Perfil de Tumaco Temperatura del Agua Fig.No.21 - Perfil de Tumaco Salinidad del Agua
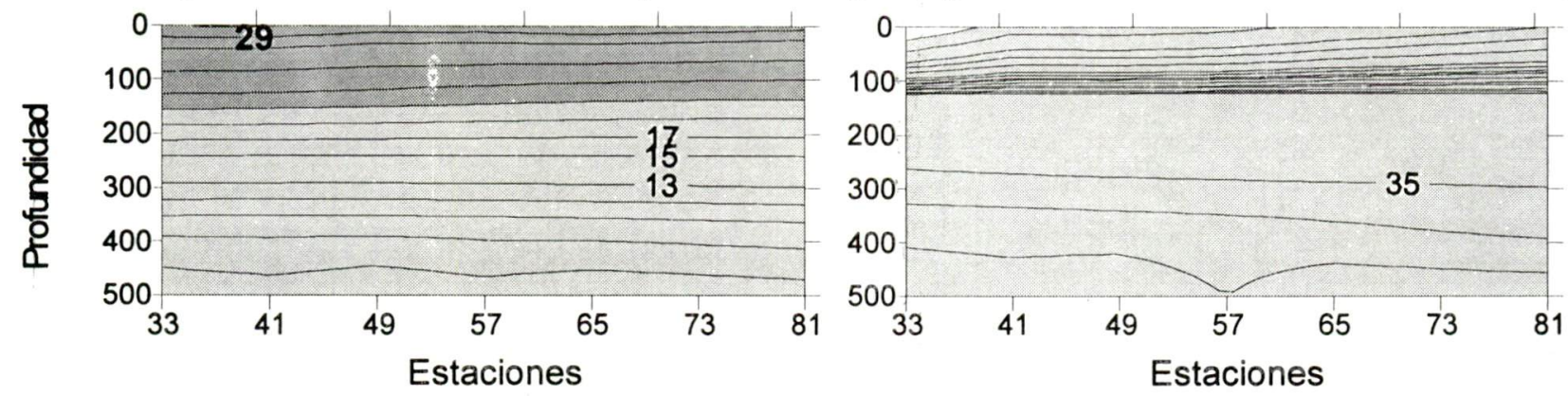

Fig.No.22 - Perfil de Gorgona Temperatura del Agua'Fig.No.23 - Perfil de Gorgona Salinidad del Aguya
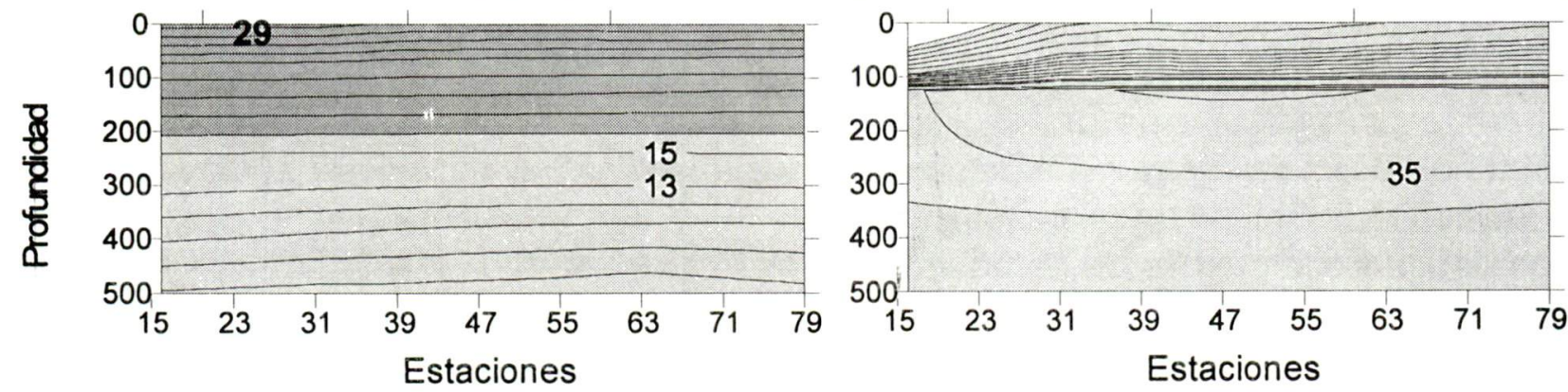
Fig.No.24 - Perfil de Buenaventura Temperatura del AfgigANo.25 - Perfil de Buenaventura Salinidad del Agua
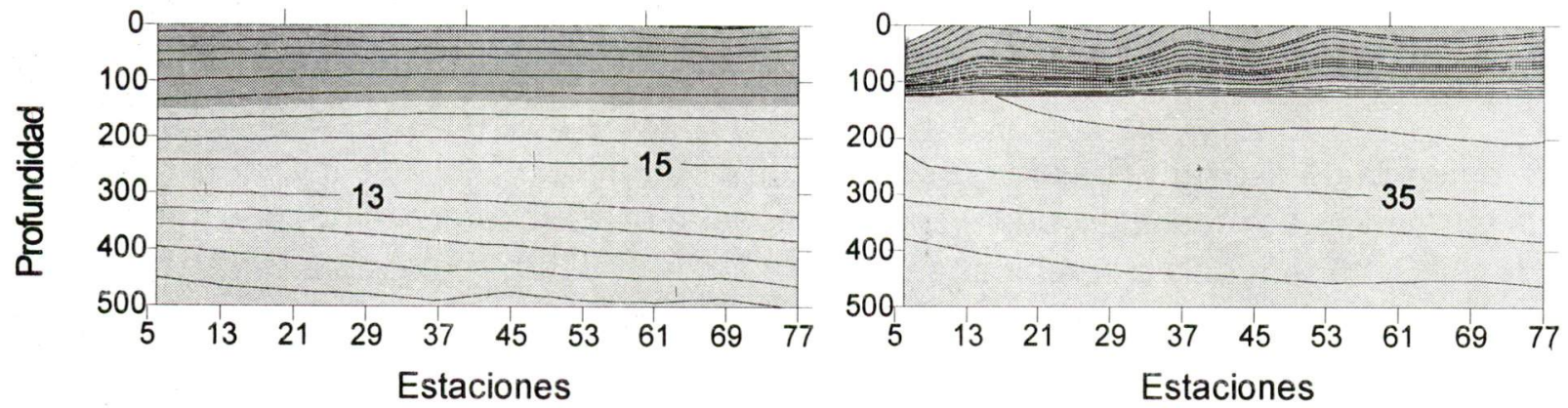

Analizando el comportamiento termohalino de la Subcorriente Ecuatorial, sobre el Pacifico Sur Colombiano, durante las condiciones del desarrollo del fenómeno de El Niño, se podría afirmar que el calentamiento que se observa a nivel superficial, incide sobre la Subcorriente, ya que las condiciones térmicas muestran los valores tipicos de esta $\left(13^{\circ} \mathrm{C}\right.$ a $\left.15^{\circ} \mathrm{C}\right)$, en una capa muy tenue entre los 250 y 300 metros, lo cual no ocurre en los periodos de 1996, ni en mayo/97.

\section{Comportamiento de la Circulación en el Pacífico Sur Colombiano.}

Las características de la circulación se realizaron, por medio del modelo geostrófico elaborado por Helland - Hansen, basándose en la teoría de la circulación de Bjerknes. La profundidad en metros fue considerada numéricamente igual a la presión en decibares (db). Para determinar el desplazamiento de la Subcorriente Ecuatorial sobre el Pacífico Sur Colombiano, se elaboraron gráficas a los 100 y $200 \mathrm{mts}$ de profundidad, en los periodos junio - octubre de 1996 y mayo - diciembre de 1997.

\section{Características de la Circulación de la Corriente a los 100 y 200 metros durante La Niña.}

\section{Junio de 1996}

La Figura No.26, presenta la circulación geostrófica de la corriente a 100 metros con referencia a $400 \mathrm{db}$, en el Pacífico Colombiano durante junio/96. A esta profundidad la corriente de Cromwell entra sobre la cuenca del Pacífico Colombiano en el sector limítrofe con aguas Ecuatorianas de coordenadas $82^{\circ} \mathrm{W}$ y $1^{\circ} 30^{\prime} \mathrm{N}-2^{\circ} 00^{\prime} \mathrm{N}$. La Subcorriente se desplaza del sector suroeste (aguas oceánicas), hacia el sector noreste (aguas costeras), atravesando prácticamente toda la cuenca del Pacífico Colombiano. La corriente de Cromwell se dirige sobre el perfil de Tumaco $\left(2^{\circ} \mathrm{N}\right)$, tomando rumbo sobre el perfil de Isla Gorgona $\left(3^{\circ} \mathrm{N}\right)$, en este punto la corriente se divide en dos ramales: el primero continua su trayectoria hacia el perfil de Buénaventura, siguiendo hacia los $5^{\circ} \mathrm{N}$ y viendo absorbido su desplazamiento por las corrientes que hacen su ingreso en el sector norte de la cuenca, debido a la influencia del brazo de la corriente de California que se desplaza sobre el litoral de Centroamérica y entra en la región norte del Pacífico Colombiano y del Panamá Bight. El segundo ramal se dirige paralelo a Gorgona $\left(3^{\circ} \mathrm{N}\right)$, y vira hacia el sur desplazándose paralelo a la costa, formando una circulación anticiclónica en el sector sur del Pacífico Colombiano.

La figura No.27, corresponde a la circulación de la corriente a los 200 metros, presentándose un cambio a lo registrado a los 100 metros. Sobre el perfil de Tumaco se localiza un núcleo anticiclónico entre $1^{\circ} 30^{\prime} \mathrm{N}$ y $2^{\circ} 30^{\prime} \mathrm{N}$, viendo obstruido su desplazamiento hacia la costa, por el comportamiento que se registra de la corriente de norte a sur desde 
Fig No 26

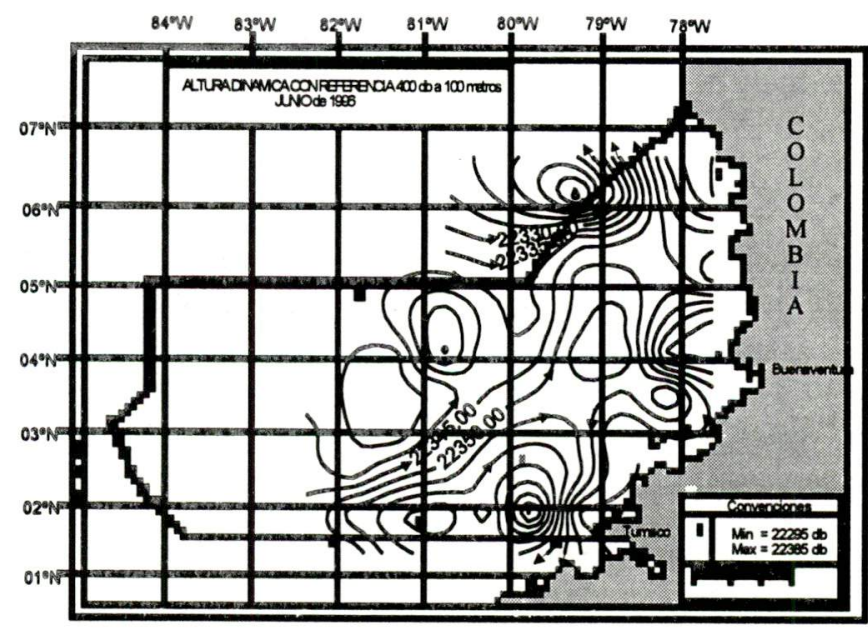

Cabo Corrientes $\left(5^{\circ} \mathrm{N}\right)$ hasta aguas limitrofes con el Ecuador. Observando las caracteristicas de la corriente, vemos cómo ésta entra sobre los $5^{\circ} \mathrm{N}$ (aguas oceánicas), dirigiéndose paralela a este perfil, tomando rumbo sobre el litoral Pacífico Colombiano Sur. Al interior de este movimiento se forma un gran núcleo anticiclónico entre los perfiles de Gorgona $\left(3^{\circ} \mathrm{N}\right)$ y Buenaventura $\left(4^{\circ} \mathrm{N}\right)$ y entre los $82^{\circ} \mathrm{W}$ a $80^{\circ} \mathrm{W}$. Estos movimientos hacen que la corriente de Cromwell no se defina muy bien a esta profundidad.

\section{Octubre de 1996.}

Analizando la topografía dinámica a 100 metros (Fig.No.28) durante octubre de 1996, vemos que la subcorriente ecuatorial influye solamente sobre el sector sur del Pacífico Colombiano ( $1^{\circ} 30^{\prime} \mathrm{N}$ y $2^{\circ} 20^{\prime} \mathrm{N}$ ), desplazándose paralelamente en el perfil de Tumaco y dirigiéndose hacia el sur en los paralelos $81^{\circ}$ Wy $81^{\circ} 30^{\prime} \mathrm{W}$.

El sector central y norte de la cuenca se ven influenciados por las corrientes que se forman en el Panamá Bight, impidiendo el ingreso de la subcorriente a los perfiles de Gorgona y Buenaventura. Este comportamiento de la corriente esta relacionado con
Fig $N^{\circ} 27$

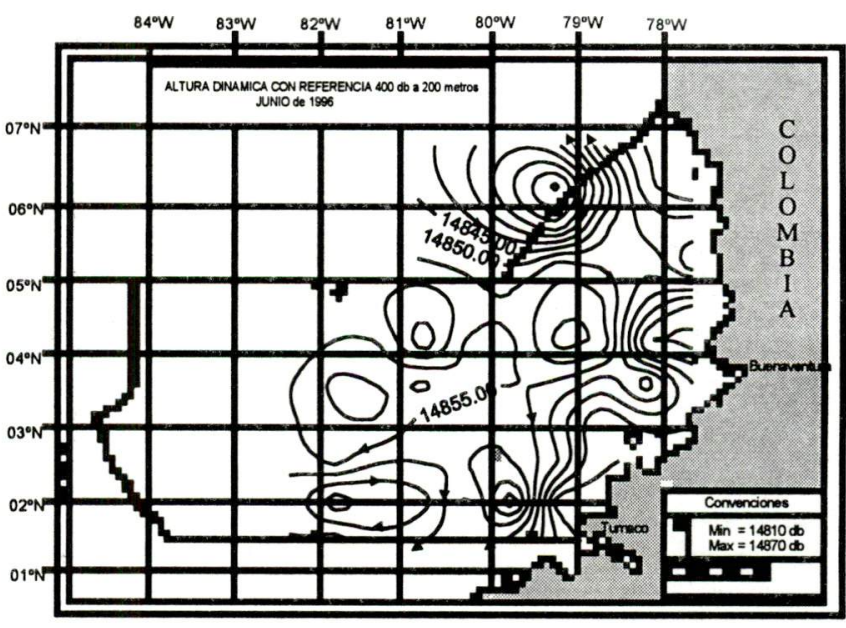

la influencia del fenómeno de La Niña, que durante la época se hacía presente sobre aguas del Pacífico Colombiano, indicando que bajo la influencia de un fenómeno de esta naturaleza las características dinámicas cambian en relación a lo que se registraba con condiciones cuasinormales o del inicio del fenómeno de La Niña (ver 3.2.1).

El comportamiento de la topografía dinámica a los 200 metros ( Fig.No.29), presenta unas caracteristicas muy similares en la parte central y norte del Pacífico Colombiano, a lo visto a los 100 metros. Sobre el sector sur la Subcorriente entra sobre el perfil de Tumaco $\left(2^{\circ} \mathrm{N}\right)$ y se dirige con trayectoria hacia la costa, para luego tomar rumbo paralelo al litoral desplazándose de sur a norte, pasando por los perfiles de Gorgona $\left(3^{\circ} \mathrm{N}\right)$ y Buenaventura $\left(4^{\circ} \mathrm{N}\right)$. Un brazo de mayor envergadura se dirige hacia la costa entre los $4^{\circ} \mathrm{N}$ y $5^{\circ} \mathrm{N}$, y el otro brazo se dirige paralelo al litoral Chocoano, tomando rumbo al Panamá Bight, para hacer su retorno hacia el Pacifico Central Colombiano. 
Fig N ${ }^{\circ} 28$

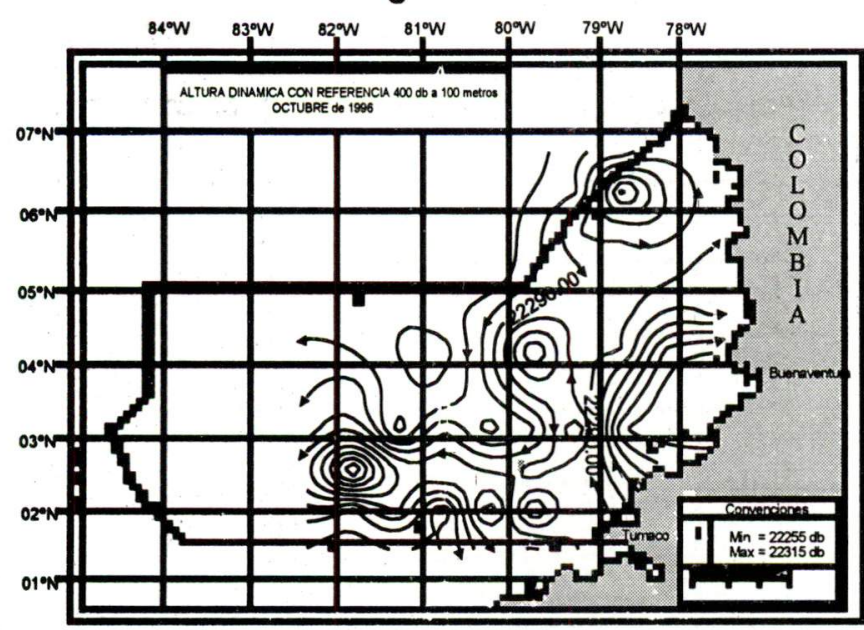

Características de la Circulación de la Corriente a los 100 y 200 metros durante El Niño.

\section{Mayo de 1997}

La circulación de la corriente durante mayo/ 97 (Fig.No.30), en la Cuenca del Pacífico Colombiano, presenta una similitud a lo observado en junio/96 (ver 3.2.1). La Subcorriente hace su ingreso en el área de estudio, en el sector sur, se desplaza en sentido suroeste hacia $\in I$ noreste, atraviesa transversalmente la cuenca y se divide en dos ramales sobre los $4^{\circ} 30^{\prime} \mathrm{N}$ : el primer ramal; más débil, es absorbido por las corrientes que se forman en el norte, desplazándolo hacia el Panamá Bight. El segundo ramal, por la acción de las fuertes corrientes del norte, vira al sur en los $79^{\circ} \mathrm{W}$, para continuar su recorrido paralelamente al litoral, pasando sobre Buenaventura y llegando a Tumaco, para tomar rumbo a aguas Ecuatorianas. Analizando dicho recorrido se determina una formación anticiclónica en su interior, de unas 120 millas de diámetro.

En mayo/97 (Fig.No.31), a los 200 metros, la circulación sufre un cambio mas demarcado, mostrando una formación anticiclónica de gran magnitud sobre la parte central y sur del área de estudio $\left(5^{\circ} \mathrm{N}\right.$ a $3^{\circ} \mathrm{N}$ y $82^{\circ} \mathrm{W}$ a $79^{\circ}$
Fig $\mathrm{N}^{\circ} 29$

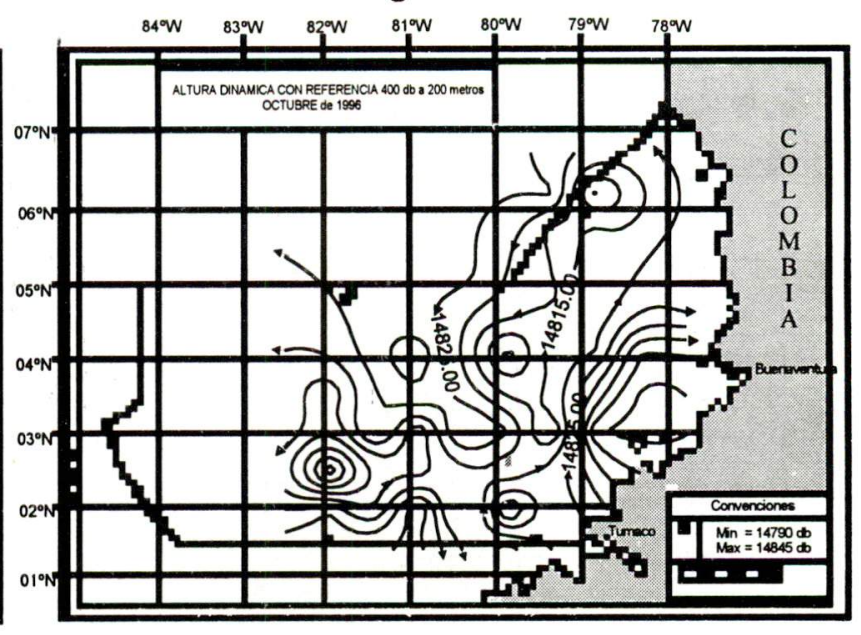

W). Analizando esta circulación anticiclónica, vemos que la corriente entra sobre los $5^{\circ} \mathrm{N}$ y $82^{\circ} \mathrm{W}$, y se dirige latitudinalmente hacia la costa, virando al sur en los $79^{\circ} \mathrm{W}$, desplazándose longitudinalmente hasta el perfil de Tumaco, para internarse posteriormente en aguas Ecuatorianas. Con este movimiento la Subcorriente no se presenta muy bien definida a esta profundidad.

\section{Diciembre de 1997}

Durante el período de diciembre/97 (Fig: No.32), a los 100 metros se aprecia cómo la subcorriente hace su ingreso sobre el sector de Tumaco, desplazándose sobre dicho perfil, para luego tomar dos rumbos diferentes: el primero hacia el sector sur con aguas ecuatorianas, formando una circulación ciclónica, y el segundo pasando sobre el perfil de Gorgona y Buenaventura, para finalmente dirigirse a la costa Chocoana. Durante este periodo se hallaba el fenómeno de El Niño en su etapa de desarrollo.

La figura No.33, representa el esquema de la corriente a los 200 metros, observándose entre $1^{\circ} 30^{\prime} \mathrm{N}$ y $2^{\circ} \mathrm{N}$ la Subcorriente dirigiéndose del sector costero hacia la zona oceánica, abarcando todo el perfil de Tumaco, para luego mezclarse con aguas provenientes del Ecuador. 
Fig $\mathrm{N}^{\circ} 30$

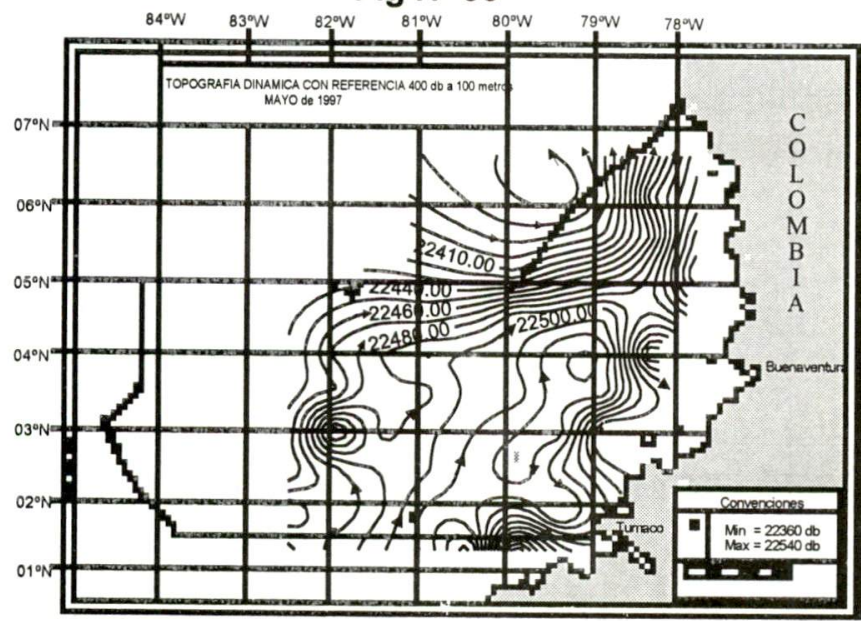

Fig $N^{0} 32$

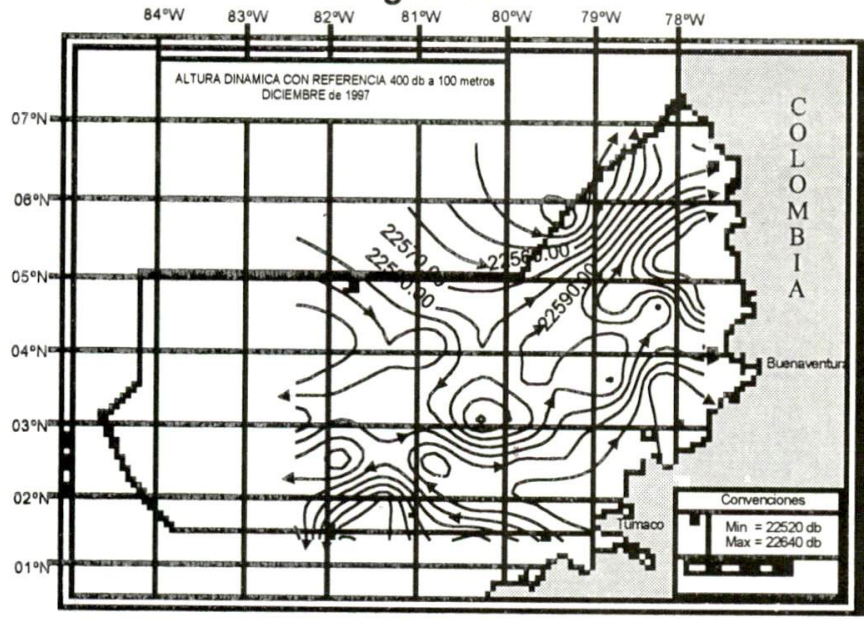

Fig $N^{\circ} 31$

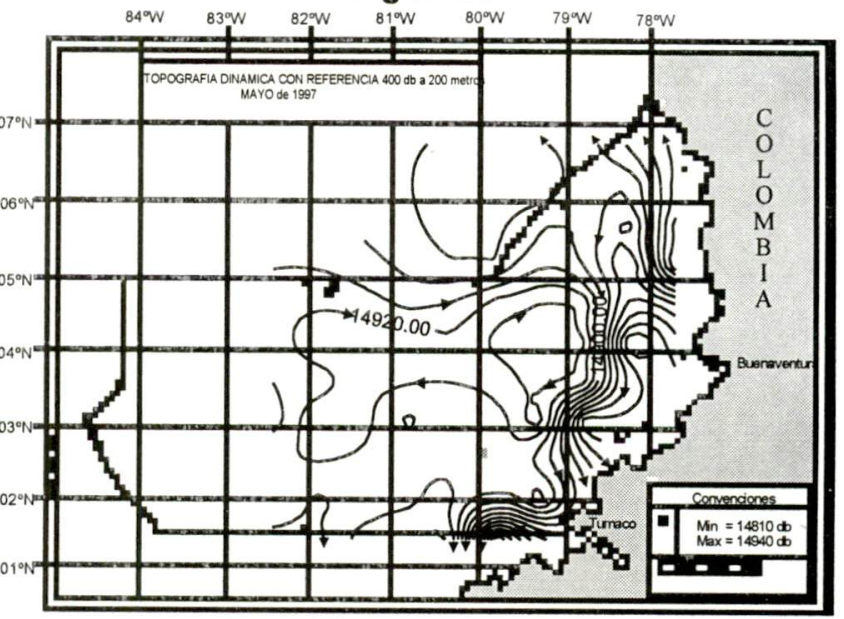

Fig No 33

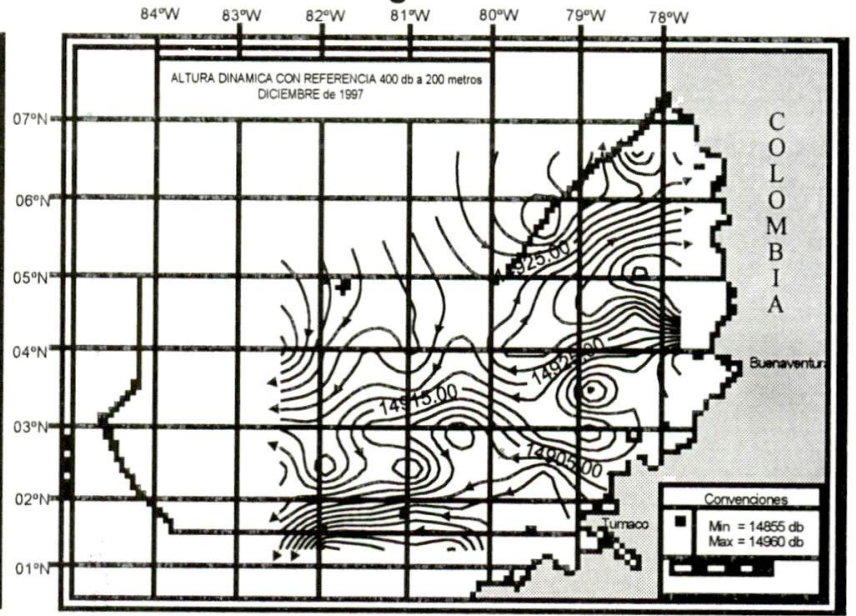

\section{CONCLUSIONES}

En el año de 1996, durante las condiciones del fenómeno de La Niña, la Corriente de Cromwell se presentó con sus índices termohalinos típicos. Sobre los diferentes perfiles la subcorriente se localizó entre las profundidades de 75 a 200 metros aproximadamente. Estas características termohalinas son las que han permitido estudiar la Subcorriente, y establecer su presencia en el área de estudio. El comportamiento de la circulación de la corriente para esta época, evidenció la presencia de la Corriente de Cromwell sobre el Pacifico Sur Colombiano, y al llegar sobre el sector central y norte de la cuenca, se ve afectada por factores hidrodinámicos, que no le permite presentarse con sus condiciones propias, como lo hace en el Pacífico Ecuatorial.

Durante la presencia del fenómeno de El Niño en el año de 1997, la Subcorriente Ecuatorial, se presentó un poco más profunda entre los 125 y 300 metros aproximadamente. La profundización de la corriente se debió al calentamiento superficial y al aumento de la salinidad. Las condiciones térmicas de la corriente durante diciembre/97 variaron, registrando los valores de temperatura de $13^{\circ}$ a $15^{\circ} \mathrm{C}$, en una capa muy tenue, (250 a 300 metros), e incidiendo solamente sobre el perfil de Tumaco, mostrando en toda la cuenca del Pacífico Colombiano a la Subcorriente afectada en su desplazamiento, como consecuencia de movimientos que obstruyen su circuiación libremente, sobre el área de estudio. 
El método geostrófico, mostró una similitud en la circulación, tanto para el periodo de fin de año (octubre - diciembre), en donde se observa a la subcorriente un poco más débil, y afectada por los movimientos de los fuertes flujos del noroeste y oeste, y para el periodo de mitad de año (mayo - junio), en donde la Subcorriente se registra un poco más fuerte, abarcando gran parte del área de estudio.

\section{BIBLIOGRAFÍA}

BUBHOV, B.A. "Circulación del Agua en la Zona Ecuatorial de los Océanos", Leningrado: Nauka, 1991.

COLIN, S.R. " EI Niño", 1996.

DARONIN, P. " Dinámica del Océano", Leningrado: Hidrometeoizdat, 1980, pp 13 - 37

EGOROV, N.I. "Oceanografía Física" Leningrado: Hidrometeoizdat, 1983, pp 406 - 435

GIRALDO, L. 1994, "Análisis de las Masas de Agua y Control de la Información Oceanográfica". Cartagena de Indias: Boletín Científico No.15 Centro de Investigaciones Oceanográficas e Hidrográficas, 1994, pp.il.

GRANT, M. " Principles of Oceanography ". USA: Prentice Hall, 1993, pp.230 - 250

LUCERO, M y CORNEJO - RODRIGUEZ, P. "Evidencia de la Corriente de Cromwell entre $92^{\circ} \mathrm{W}$ y $84^{\circ} \mathrm{W}, \quad$ observada en periodos normales y durante El Niño 82 - $83^{\prime \prime}$. Ecuador: Inocar, 1990.

MILLAN, E. y BEJARANO, J. " Pacífico Colombiano Condiciones Termohalinas Estandarización

de la Curva T-S ". Cartagena de Indias: Boletín Científico No. 15 Centro de Investigaciones Oceanográficas e Hidrográficas. 1994, 39 pp.il.

OCHOA, N y B. ROJAS DE MENDIOLA. " Identificación del Fenómeno El Niño, a través de los organismos fitoplanctónicos ". 1994.

PHINLANDER, S.G. "EI Niño, La Niña, and the Southern Oscillation". San Diego: Academic Press, inc, 1990.

PINEDA, A. " Condiciones Hidrológicas en la Cuenca del Pacífico Colombiano. Tumaco: Boletín Científico Centro Control Contaminación del Pacífico ", 1995. 25 pp.il.

POVEDA, G. Y MESA, O. "Las Fases Extremas del Fenómeno ENSO (EI Niño y La Niña) y su

Influencia Sobre la Hidrología de Colombia ". Mexico: Ingeniería Hidráulica Vol. XI. Numero 1, 1996, pp 21-37.

SMIRNOV, G.N. "Oceanología", Moscú: Nauka, 1974

SUJAVIEY, V.F. "Régimen Hidrológico en los Océanos Atlántico y Pacífico", Kiev: UMK, 1992. pp $113-205$ 